\title{
Inhalt
}

Abbildungs- und Tabellenverzeichnis

Den Teufel des Traumas mit Beelzebub austreiben?

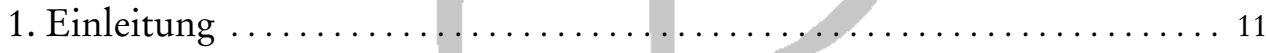

1.1 Danksagung. ..................................... 15

2. Grundlagen der Psychotraumatologie. . . . . . . . . . . . . . . 17

2.1 Zur Geschichte der Psychotraumatologie .................... 17

2.1.1 Psychotraumatologie als Forschungs- und Praxisfeld . . . . . . . . . . . 23

2.2 Psychische Traumatisierung - eine Begriffsklärung .............. 26

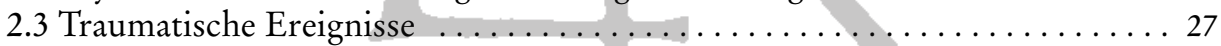

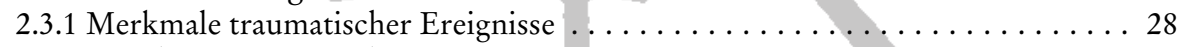

2.3.2 Typologie traumatischer Situationen. . . . . . . . . . . . . . . 31

2.4 Verlaufsmodell psychischer Traumatisierung ................... 34

2.5 Die peri- und posttraumatische Stressphysiologie ................ 35

2.5.1 Neurobiologische Aspekte . . . . . . . . . . . . . . . . . . . . . . . . . 37

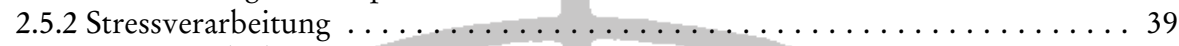

2.5.3 Traumagedächtnis.............................. 42

2.6 Folgen und Auswirkungen psychischer Traumatisierung . . . . . . . . 45

2.6.1 Formen von Gedächtnisstörungen und Dissoziation ............. 47

2.6.2 Auswirkungen früher Gewalterfahrungen auf die Bindungsfähigkeit . . . . . 50

2.7 Diagnostik der Traumafolgestörungen................... 51

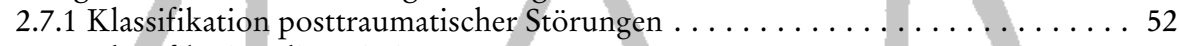

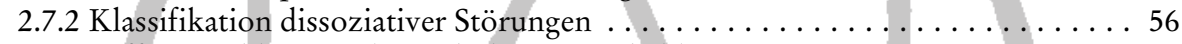

2.7.3 Differenzialdiagnostik Borderlinepersönlichkeitsstörung

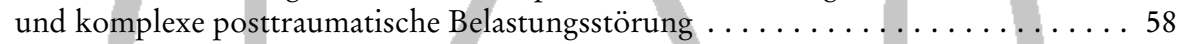

2.7.4 Strukturelle Interviews und Fragebögen zur Erstellung der Diagnose. . . . . . . 60

2.8 Themen aus der speziellen Psychotraumatologie . . . . . . . . . . . . . 61

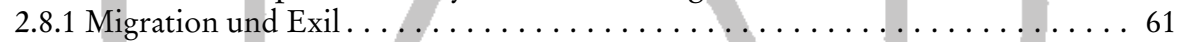

2.8 .2 Sexueller Kindesmissbrauch $\ldots \ldots \ldots \ldots \ldots \ldots \ldots \ldots \ldots \ldots \ldots \ldots \ldots$

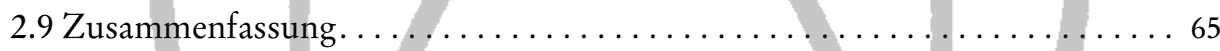

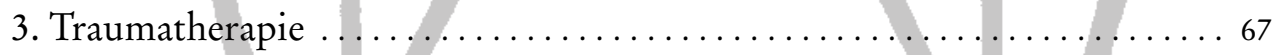

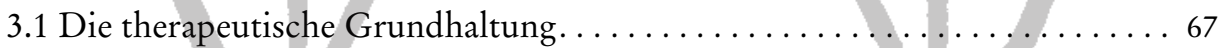

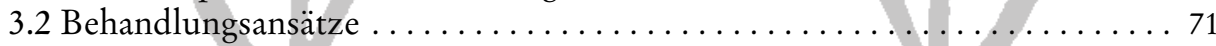

3.2.1 Psychotraumatologisch fundierte Psychotherapie (PFP) . . . . . . . . 72

3.2.2 Das Phasenmodell .............................. 74

3.2.3 Traumatherapeutische Verfahren nach dem Phasenmodell ............ 75

3.2.4 Die Orientierungsphase $\ldots \ldots \ldots \ldots \ldots \ldots \ldots \ldots \ldots \ldots \ldots \ldots \ldots$

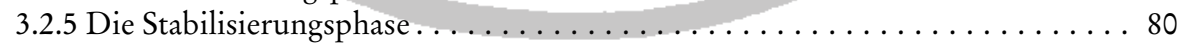

3.2.6 Verfahren und Techniken innerhalb der Stabilisierungsphase . . . . . . . . . 81

3.2.7 Ziele der Stabilisierungsphase . . . . . . . . . . . . . . . . . . . . . 94

3.2.8 Untersuchungen von ambulanten und stationären Behandlungen

mit komplex traumatisierten Patientinnen. . . . . . . . . . . . . . . . . . 95 
4. Musiktherapie und Psychotraumatologie.................. 97

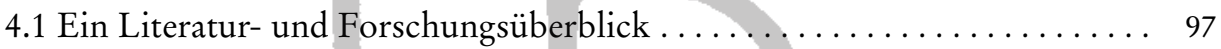

4.2 Musiktherapeutische Behandlungsansätze .................... 101

4.2.1 Beispiele musik-traumatherapeutischer Handlungsmodelle . . . . . . . . . 103

4.3 Musiktherapie als traumaadaptierter Behandlungsansatz............ 105

4.3.1 Die musikalische Beziehungsgestaltung . . . . . . . . . . . . . . . 106

4.3 .2 Das Setting ..................................... 109

4.3.3 Stabilisierung durch Ressourcenaktivierung ................. 113

4.3.4 Schonende Traumabearbeitung durch Symbolisierung . . . . . . . . . . . 117

4.3.5 Beispiele traumaadaptierter musiktherapeutischer Techniken ........... 117

4.3.6 Ziele im traumaadaptierten Behandlungsansatz . . . . . . . . . . . . . . . 124

5. Musiktherapie mit traumatisierten Erwachsenen:

Vier Fallrekonstruktionen aus der klinischen Praxis $\ldots \ldots \ldots \ldots \ldots \ldots . \ldots 125$

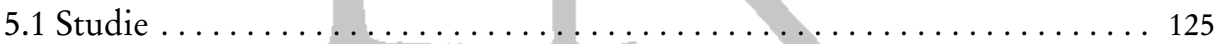

5.1 .1 Klinische Voraussetzungen............................ 125

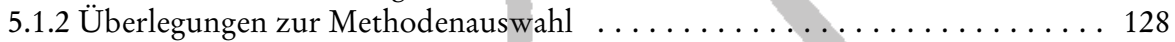

5.1 .3 Mixed Methods .................................. 129

5.2 Qualitative Datensammlung, Erhebung und Aufbereitung ........... 131

5.2 .1 Qualitative Inhaltsanalyse. . . . . . . . . . . . . . . . . . . . . 131

5.2.2 Kategorisierung von traumabedingten und musikbedingten

Faktoren - Deduktive Kategorienanwendung. .................... 133

5.2.3 Ablaufmodell für die inhaltsanalytische Auswertung $\ldots \ldots \ldots \ldots \ldots . . \ldots 140$

5.2.4 Inhaltsanalytische Auswertung. ...................... 143

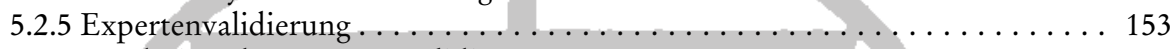

5.2.6 Ergebnisse der Expertenvalidierung . ....................... 154

5.2.7 Beurteilung der qualitativen Inhaltsanalyse. $\ldots \ldots \ldots \ldots \ldots \ldots \ldots \ldots \ldots$

5.3 Quantitative Datensammlung, Erhebung und Aufbereitung ......... 166

5.3.1 Vegetative Diagnostik (Biofeedbackanalyse).................... 166

5.3.2 Ergebnisse der Vegetativen Diagnostik . . . . . . . . . . . . . . . . . . . . 169

5.3 .3 SUC- und SUD-Skalen ............................. 173

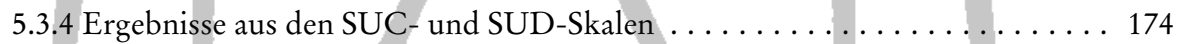

5.3.5 Die Symptomcheckliste SCL-90R . . . . . . . . . . . . . . . . . 176

5.3.6 Ergebnisse aus der Symptomcheckliste SCL-90-R . . . . . . . . . . . . 183

5.3.7 Beurteilung der quantitativen Messinstrumente ............... 188

6. Zusammenfassung. ............................ 191

7. Literaturverzeichnis . . . . . . . . . . . . . . . . . . ...... 197

Anhang .............................................. 211 


\section{Abbildungs- und Tabellenverzeichnis}

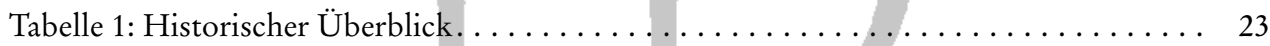

Tabelle 2: Traumahäufigkeit und Störungshäufigkeit . . . . . . . . . . . . . 29

Abbildung 1: Anzahl von Traumatisierungen und PTSD-Prävalenzen . . . . . . . . . . 29

Abbildung 2: Schützende und belastende Faktoren $\ldots \ldots \ldots \ldots \ldots \ldots \ldots \ldots \ldots$

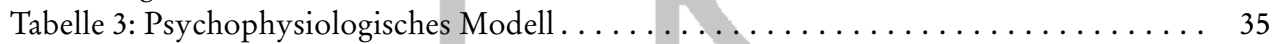

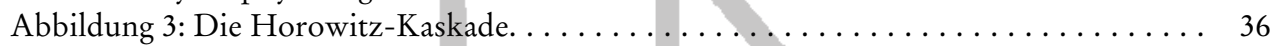

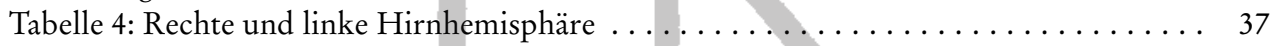

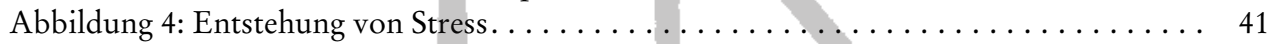

Tabelle 5: Neurohormone bei Psychotraumata. . . . . . . . . . . . . . . 42

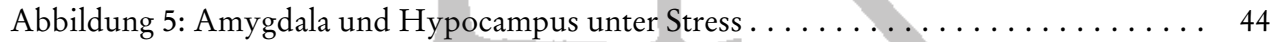

Tabelle 6: Phylogenetische Stadien der Polyvagaltheorie. . . . . . . . . . . . . . . 47

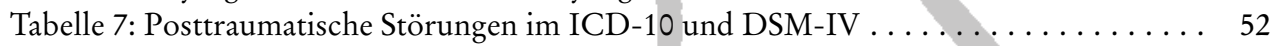

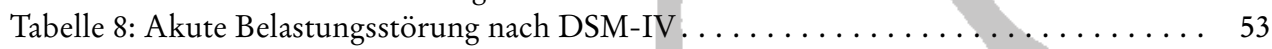

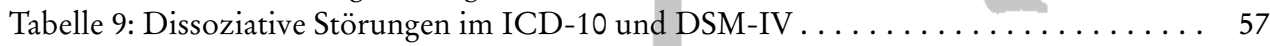

Tabelle 10: BPS und komplexe PTBS nach DESNOS . . . . . . . . . . . . . . . 59

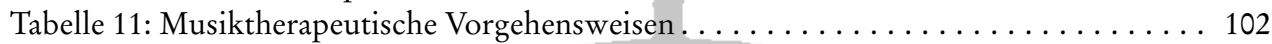

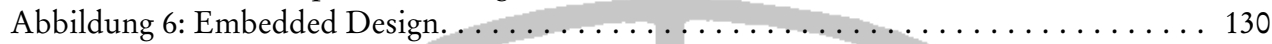

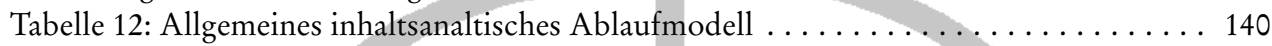

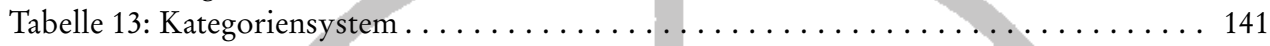

Tabelle 14: Qualitative Inhaltsanalyse Patientin A $\ldots \ldots \ldots \ldots \ldots \ldots \ldots \ldots \ldots \ldots \ldots$

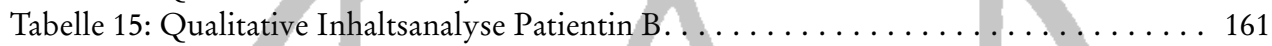

Tabelle 16: Qualitative Inhaltsanalyse Patient C . . . . . . . . . . . . . 162

Tabelle 17: Qualitative Inhaltsanalyse Patient D . . . . . . . . . . . . . 163

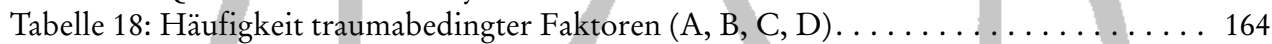

Tabelle 19: Häufigkeit musikbedingter Faktoren $(A, B, C, D) \ldots \ldots \ldots \ldots \ldots \ldots$

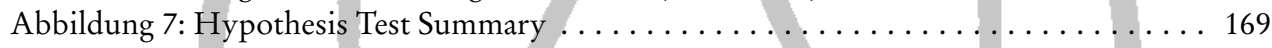

Abbildung 8: Skin Conductance Level Patientin A. . . . . . . . . . . . . . . . 170

Abbildung 9: Skin Conductance Level Patientin B . . . . . . . . . . . . . . 171

Abbildung 10: Skin Conductance Level Patient C . . . . . . . . . . . . . . . 172

Abbildung 11: Skin Conductance Level Patient D . . . . . . . . . . . . . . . . 173

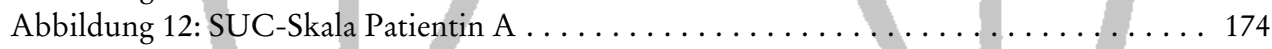

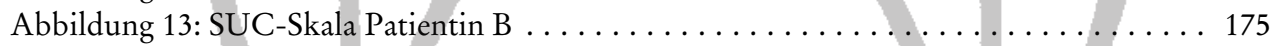

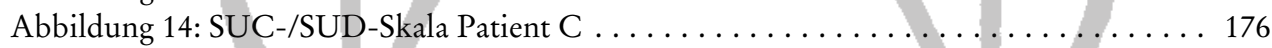

Tabelle 20: Statistisches Auswertungsblatt der SCL-90-R . . . . . . . . . . . . . . 180

Tabelle 21: Maximalgrenzen von Missings in der SCL-90-R . . . . . . . . . . . . 182

Abbildung 15: SCL-90-R Patientin A. . . . . . . . . . . . . . . . . . . . . . 183

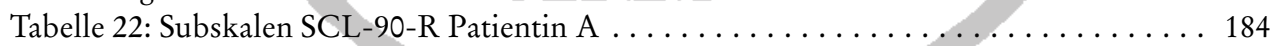

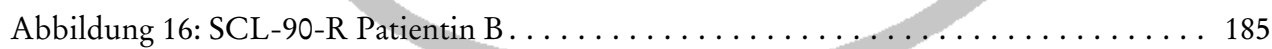

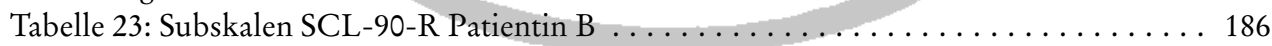

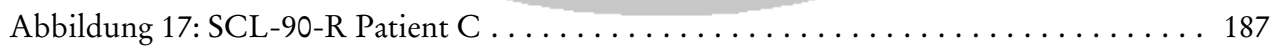

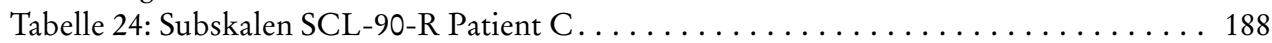

